\section{Starting with serotonin}

\section{How a high-rolling father of drug discovery repeatedly beat the odds}

Ann G. Sjoerdsma

Improbable Books. Silver Spring, Maryland, USA. 2008.

617 pp. \$27.50. ISBN: 978-0-615-16558-5 (hardcover).

Reviewed by Dennis L. Murphy

Laboratory of Clinical Science, National Institute of Mental Health (NIMH), Bethesda, Maryland, USA.

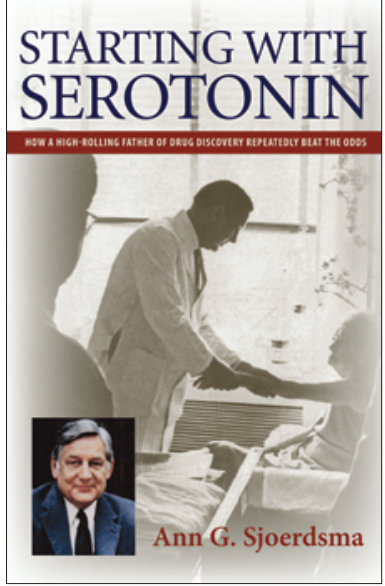

$S$ ing with serotonin chronicles the life of Albert Sjoerdsma, a major contributor to the era of clinically directed science and therapeutic drug development from the mid-1950s through the early 1990s. Written by his daughter Ann, who had access to more personal material than is typical for biographers of scientists, the book tells of Sjoerdsma's groundbreaking work in the field of clinical pharmacology, starting with the neurotransmitter serotonin. The title is mostly an opening gambit, as the book progresses to describe Sjoerdsma's contributions to our knowledge of catecholamines and antihypertensive drugs and his exploratory clinical pharmacological work in many other important areas.

Sjoerdsma's career was divided into two major phases. He began as a frontline clinical scientist, working with a "wild bunch" of clinical pharmacologists and biochemists, tackling novel problems in our understanding and treatment of a wide range of basic metabolic and clinical disorders. $\mathrm{He}$ developed into a mentor of young scientists of distinction, many of whom flocked to the NIH during the World War II draft to participate in a new wave of scientific breakthroughs and therapeutic triumphs in diverse, clinically relevant research areas. Seen by some as the "father of modern clinical pharmacology," Sjoerdsma had many successes of note. New biochemical assays fostered the understanding of collagen pathway metabolism, leading to therapeutics for disorders such as scleroderma and neglected infectious diseases like African trypanosomiasis. However, with success came more than a few mishaps and dead

The views expressed here do not necessarily represent those of the NIMH, NIH, Department of Health and Human Services, or United States Government. ends, which are honestly noted. In some ways, Ann provides a glimpse of not just a man but a scientific era. Yet in contrast to A.R. Green's historical perspective of the evolution of serotonin research (1), great scientists and clinical pharmacologists of the era outside of Sjoerdsma's immediate circle are rarely mentioned.

The second phase of Sjoerdsma's career followed his departure from the NIH after 22 years to take on the challenge of clinical drug development in the pharmaceutical industry. He initiated a research program in Strasbourg, France, hiring a large cohort of scientists to focus on creative drug development.

Pictures of colleagues and family are an exceptional addition, capturing visual elements of the era, and extensively annotated references thoroughly document Sjoerdsma's contribution to this 40-year period of creative basic science and drug development and the combative experiences (especially with the FDA) that characterized his career. This era has perhaps only been eclipsed by the recent developments in genomic and postgenomic medicine.

As someone with experience as an experimental clinical pharmacologist faced with seeking FDA approval for investigational drugs after enactment of the apparently prohibitive post-thalidomide KefauverHarris Amendment in 1962, I can sympathize with the notion that Sjoerdsma subsequently felt "handcuffed" (his word) going forward with his clinical pharmacology-based studies. This led to no limit of personal angst for Sjoerdsma; he struggled at the NIH and later as a pharmaceutical executive forced to deal with the "new" FDA.

In some respects, the book could have been more user-friendly. Its length and style of mixing anecdotes with a developed, subject-oriented format make it hard to identify many of the key achievements and the author's points. Many statements included - for example, stories of "eureka" findings revealed during Saturday morning lab meetings with younger colleagues - were likely obtained by direct interviews, but this is unclear, as they are not enclosed in traditional quotation marks.

Sjoerdsma followed a philosophy of undertaking research in multiple new directions simultaneously. He recruited young scientists of merit who were open to new ideas, choosing not to confine them to an already-developed research project. The author's similar style of discussing multiple topics side-by-side, with only the surnames of colleagues occasionally provided to link the various areas of Sjoerdsma's research, sometimes makes for difficult reading.

The book will appeal to scientists with broad clinical research interests, not just those working on serotonin. Sometimes Sjoerdsma's clever, hypothesis-based ideas (which would perhaps now be considered grant-worthy), after experimental investigation, just "never really held up" - a not unfamiliar scenario that experienced scientists have likely found themselves in; they may thus find encouragement in the fact that with Sjoerdsma's successes also came failures. There is a lot to be learned from this chronicle of this expert clinical scientist's experiences, during his periods both as a member of the "wild bunch" of NIH clinical pharmacologists and as a scientist working in the drug development industry.

1. Green, A.R. 2008. Gaddum and LSD: the birth and growth of experimental and clinical neuropharmacology research on 5-HT in the UK. Br. J. Pharmacol. 154:1583-1599. 